\title{
Valutazione degli effetti del cabozantinib nel carcinoma tiroideo differenziato radioiodio refrattario nel trial di fase 3 "COSMIC-311"
}

\author{
Carmelo Gusmano $^{1} \cdot$ Aldo E. Calogero $^{1}$
}

Accettato: 27 novembre 2021 / Pubblicato online: 3 marzo 2022

c) The Author(s) 2022, corrected publication 2022

Commento a:

Cabozantinib for radioiodine-refractory differentiated thyroid cancer (COSMIC-311): a randomised, double-blind, placebo-controlled, phase 3 trial. M.S. Brose, B. Robinson, S.I. Sherman, J. Krajewska, C.-C. Lin, F. Vaisman, A.O. Hoff, E. Hitre, D.W. Bowles, J. Hernando, L. Faoro, K. Banerjee, J.W. Oliver, B. Keam, J. Capdevila.

Lancet Oncol (2021) 22(8):1126-1138

Il carcinoma differenziato della tiroide (Differentiated Thyroid Cancer, DTC) rappresenta il 90-95\% delle nuove diagnosi di tumore tiroideo e include il carcinoma papillare $(80 \%)$, il carcinoma follicolare (10-15\%) e, meno frequentemente, le varianti istologiche a cellule di Hürtle e indifferenziate [1]. Le strategie di trattamento per il DTC sono multimodali con un approccio adattato al rischio e includono la sorveglianza attiva, la chirurgia e la radioterapia [2]. Nonostante la prognosi sia relativamente favorevole, più del $15 \%$ dei pazienti sviluppa una malattia metastatica refrattaria al radio-iodio; in tale evenienza, la prognosi diventa significativamente peggiore [1]. In questi casi, le opzioni di trattamento includono i farmaci inibitori della tirosin-chinasi (Tyrosine Kinase Inhibitors, TKI), sorafenib e lenvatinib, che agiscono su diversi target, in particolare sul recettore del fattore di crescita vascolare endoteliale (Vascular Endothelial Growth Factor Receptor, VEGFR) e altri recettori chinasici coinvolti nella proliferazione e sopravvivenza dei tumori e nell' angiogenesi. Nonostante la maggior parte dei pazienti con DTC refrattario al radioiodio ottenga un controllo della malattia con sorafenib o lenvatinib, la maggior parte svilupperà resistenza al trattamento e progressione della malattia, con una sopravvivenza globale mediana inferiore a 5 anni [2].

\footnotetext{
$\triangle$ A.E. Calogero

aldo.calogero@unict.it

1 Dipartimento di Medicina Clinica e Sperimentale, Università di Catania, Catania, Italia
}

Un recente trial clinico in fase 3, multicentrico, randomizzato, in doppio cieco, placebo-controllato, denominato "COSMIC-311" ha valutato l'impiego del cabozantinib, un TKI che riduce la crescita e l'angiogenesi dei tumori maligni, in pazienti adulti (età maggiore di 16 anni) affetti da DTC e malattia residua dopo il trattamento con $\mathrm{I}^{131}$ o che erano stati considerati non idonei al trattamento radioterapico [1]. I pazienti arruolati avevano già subito un trattamento con lenvatinib e/o sorafenib, ma avevano comunque sviluppato una progressione della malattia, valutata secondo i criteri RECIST 1.1. Il meccanismo d'azione del cabozantinib prevede l'inibizione delle vie di segnalazione cellulare mediate da VEGFR2, AXL, MET e RET, implicate nello sviluppo della resistenza tumorale alle terapie non chirurgiche ( $\mathrm{I}^{131} \mathrm{o}$ farmaci) finora approvate. Gli endpoint primari dello studio erano quelli di valutare il tasso di risposta alla terapia (objective response rate intention-to-treat, OITT), valutata secondo i criteri RECIST, in 100 pazienti selezionati in maniera random, e la sopravvivenza senza progressione della malattia (progression free-survival, PFS) in tutto il campione trattato, confrontando i pazienti che hanno assunto cabozantinib per via orale al dosaggio di $60 \mathrm{mg}$ con il gruppo di controllo. L'eventuale progressione della malattia è stata valutata attraverso metodiche di imaging (RMN o TC) all' $\mathrm{i}$ nizio del trial, ogni 8 settimane dopo la randomizzazione e ogni 12 settimane dopo i 12 mesi di trattamento.

I principali effetti collaterali legati all'impiego del farmaco sono stati l'insorgenza di eritrodisestesia palmo-plantare (13/125 pazienti trattati, $10 \%)$, ipertensione (11/125 pazienti trattati, 9\%), diarrea (9/125 pazienti, $7 \%$ ) e ipocalcemia (9/125 pazienti, 7\%). Lo studio ha evidenziato la superiorità del trattamento con cabozantinib rispetto al placebo nell'aumentare significativamente la PFS correlata anche all'aumentato tasso di stabilizzazione della malattia metastatica, superiore nel braccio del cabozantinib (54/125, 43\%) rispetto al placebo (10/62 pazienti, $16 \%)$.

I pazienti con DTC refrattario allo radioiodio, che hanno sviluppato una progressione della malattia durante il trattamento con terapia target per VEGFR o successivamente ad 
essa, possiedono poche alternative terapeutiche, che includono il lenvatinib, il sorafenib, la chemioterapia citostatica o trattamenti palliativi. I risultati del trial COSMIC-311 fanno propendere verso un utile impiego del cabozantinib, già approvato nella terapia del carcinoma midollare della tiroide, anche ai pazienti con DTC in stadio avanzato con progressione nonostante il trattamento con radioiodio o altri TKI. I risultati di questo studio confermano, inoltre, il buon profilo di sicurezza e la maneggevolezza del farmaco già sperimentato in altri trial e offrono, pertanto, un trattamento maggiormente efficace ai pazienti che, nello stadio avanzato della malattia tumorale, hanno scarse opzioni terapeutiche.

Funding Note Open access funding provided by Università degli Studi di Catania within the CRUI-CARE Agreement.

Open Access This article is licensed under a Creative Commons Attribution 4.0 International License, which permits use, sharing, adaptation, distribution and reproduction in any medium or format, as long as you give appropriate credit to the original author(s) and the source, provide a link to the Creative Commons licence, and indicate if changes were made. The images or other third party material in this article are included in the article's Creative Commons licence, unless indicated otherwise in a credit line to the material. If material is not included in the article's Creative Commons licence and your intended use is not permitted by statutory regulation or exceeds the permitted use, you will need to obtain permission directly from the copyright holder. To view a copy of this licence, visit http://creativecommons.org/licenses/by/4. $0 \%$

\section{Bibliografia}

1. Brose MS, Robinson B, Sherman SI et al (2021) Cabozantinib for radioiodine-refractory differentiated thyroid cancer (COSMIC311): a randomised, double-blind, placebo-controlled, phase 3 trial. Lancet Oncol 22:1126-1138

2. Haugen BR, Alexander EK, Bible KC et al (2016) 2015 American Thyroid Association management guidelines for adult patients with thyroid nodules and differentiated thyroid cancer: the American Thyroid Association Guidelines Task Force on Thyroid Nodules and Differentiated Thyroid Cancer. Thyroid 26(1):1-133

Nota della casa editrice Springer Nature rimane neutrale in riguardo alle rivendicazioni giurisdizionali nelle mappe pubblicate e nelle affiliazioni istituzionali. 